\title{
Visual Storylines: Semantic Visualization of Movie Sequence
}

\author{
Tao Chen ${ }^{1}$, Ai-Dong Lu ${ }^{2}$, Shi-Min $\mathrm{Hu}^{1}$ \\ ${ }^{1}$ TNList, Department of Computer Science and Technology, \\ Tsinghua University, Beijing 100084, China \\ ${ }^{2}$ Department of Computer Science, University of North Carolina at Charlotte, USA
}

\begin{abstract}
This paper presents a video summarization approach that automatically extracts and visualizes movie storylines in a static image for the purposes of efficient representation and quick overview. A new type of video visualization, Visual Storylines, is designed to summarize video storylines in a succinct visual format while preserving the elegance of original videos. This is achieved with a series of video analysis, image synthesis, relationship quantification and geometric layout optimization techniques. Specifically, we analyze video contents and quantify video story unit relationships automatically through clustering video shots according to both visual and audio data. A multi-level storyline visualization method then organizes and synthesizes a suitable amount of representative information, including both locations and interested objects and characters, with the assistants of special visual languages, according to the relationships between video story units and temporal structure of the video sequence. Several results have demonstrated that our approach is able to abstract the storylines of professionally edited video such as commercial movies and TV series. Preliminary user studies have been performed to evaluate our approach and the results show that our approach can be used to assist viewers to grasp video contents efficiently, especially when they are familiar with the context of the video, or a text synopsis is provided.
\end{abstract}

Keywords: Video Summarization, Video Visualization, Geometric Layout.

\section{Introduction}

In recent years, both the quality and quantity of digital videos ${ }^{29}$ have been increasing impressively with the development of visual media technology. A vast amount of movies, TV programs ${ }^{3}$ and home videos are being produced every year for various entertainment or education purposes. Under such circumstances, video summarization techniques are desperately required for ${ }^{34}$ the video digestion and filtering process by providing viewers an efficient tool to understand video storylines without watching the entire video sequence.

Currently, existing video summarization methods mainly fo- ${ }_{38}^{38}$ cus on news programs or home videos, which usually contain simple spatiotemporal structures and straightforward storylines. Those methods cannot successfully handle professionally edited movies and TV programs, where directors tend to use more sophisticated screen techniques. For example, a movie may ${ }^{43}$ have two or several storylines alternately depicted in an irregular sequence. Also, technically, many existing methods summarize a video sequence with collections of key frames or regions 46 of interest (ROIs) without high-level information such as loca- 47 tion and occurrence. We believe that these information should 48 be carefully embedded in the video analysis and summarization 49 process.

Our goal is to present a visually pleasing and informative ${ }_{51}$ way to summarize the storylines of a movie sequence in one 52 static image. There are many advantages of using a still image ${ }_{53}$ to summarize a video sequence $[1,2,3,4,5]$, since an image 54
28 is generally much smaller and easier for viewers to understand. The methods that use still images to visualize video clips can be classified into two types according to their applications. One is to visualize a short video clip, mainly focus on one or two characters and their spatial motion, e.g. $[6,7]$; the other is to visualize a related longer video clip that is capable of telling a semantic story, e.g. $[1,2,3,5]$. Our method belongs to the later. A common problem for this type of methods is that due to the highly compact form and losses of information (e.g. audio, text and motion), it's nearly impossible for viewers to extrac$t$ the underlining stories without being aware of the context of the video or appropriate text descriptions. Even with this information provided, using previous methods is still very hard to recover the sophisticated storylines since they are lack of analysis of scene relations. We believe that by properly considering vision and audio features and carefully designing visualization form, such a semantically difficult problem can be tackled for a good many of professionally edited movies and TV programs.

In this paper, we present a new Visual Storylines method to assist viewers to understand important video contents by revealing essential information of video story units and their relationships. Our approach can produce a concise and visually pleasing representation of video sequences, which highlights most important video contents and preserves the balance coverage of original sequences. Accompanying the original text description of videos (plots), these results assist viewers to understand video topics and select their desired ones without watching all 
of them. Specifically, we first present an automatic video analy-109 sis method to extract video storylines by clustering video shot-110 $\mathrm{s}$ according to both visual and audio data. We also design $\mathrm{a}_{11}$ multi-level visual storyline method to visualize both abstract ${ }_{112}$ story relationships and important video segments. We have de-113 signed and performed preliminary user studies to evaluate our ${ }_{114}$ approach and collected very encouraging results.

The main contribution of our approach is a series of automat-116 ic video analysis, image synthesis, and relationship quantifica-117 tion and visualization methods. We have seamlessly integrated ${ }_{118}$ techniques from different fields to produce an highly compact 119 summary of video storylines. Both the results and evaluation ${ }_{120}$ demonstrate that our approach exceeds previous methods by 121 highlighting important video contents and storylines from pro-122 fessionally edited movies and TV programs.

The remainder of this paper is organized as follows. We first 124 summarize related video summarization, analysis and represen-125 tation approaches in Section 2. Section 3 presents our automat-126 ic approach to analyzing video structures and extracting story-127 lines. Section 4 describes our multi-level storyline visualization 128 method that significantly enriches abstract storylines through a129 series of video analysis and image synthesis methods. We de-130 scribe and discuss our user studies to evaluate our approach and ${ }_{131}$ provide experimental results in Section 5. Finally, Section $6_{132}$ concludes the paper.

\section{Related Work}

Our work is closely related to video summarization, which ${ }_{138}$ has been an important research topic in the fields of Computer ${ }_{139}$ Vision, Multimedia and Graphics. Video summarization ap- ${ }_{140}$ proaches often focus on content summarization [8]. A good ${ }_{141}$ survey of both dynamic and static video summarization meth-142 ods has been provided by Huet and Merialdo [9]; in which they ${ }_{143}$ also presented a generic summarization approach using $\mathrm{Max}^{-144}$ imum Recollection Principle. Very recently, Correa et al. [6] $]_{145}$ proposed dynamic video narratives, which depicted motions of ${ }_{146}$ one or several actors over time. Barnes et al. [10] present-147 ed Video Tapestries which summarized video in the form of $\mathrm{a}_{148}$ multiscale image, where users can interactively view the sum-149 marization of different scales with continuous temporal zoom.150 These two methods represent state-of-the-art of dynamic summarization.

In this paper we concentrate on approaches of static visual representations, which require synthesis of image segments ex-152 tracted from a video sequence. For example, the video booklet ${ }_{153}$ system [1] proposed by Hua et al. selected a set of thumbnails 154 from original video and printed them out on a predefined set of ${ }_{155}$ templates. Although this approach achieved a variety of form-156 $\mathrm{s}$, the layout of predefined booklet templates was usually not ${ }_{157}$ compact. Stained-glass visualization [2] was another kind of ${ }_{158}$ highly condensed video summary technique, in which selected ${ }_{159}$ key-frames with an interesting area were packed and visual-160 ized using irregular shapes like a stained-glass. Different from ${ }_{161}$ this approach, this paper synthesizes images and information ${ }_{162}$ collected from video sequences to produce smooth transitions ${ }_{163}$ between images or image ROIs. Yeung et al. presented a pictorial summary of video content [3] by arranging video posters in a timeline, which summarized the dramatic incident in each story unit. Ma and Zhang [4] presented a video snapshot approach that not only analyzed the video structure for representative images, but also used visualization techniques to provide an efficient pictorial summary of video. These two approaches showed that key frame based representative images were insufficient to recover important relations in a storyline. Among all forms of video representations, Video Collage [5] was the first to give a seamlessly integrated result. Different from their technique, our approach reveals the information of locations and relations between interested objects and preserves important storylines.

This paper is also related to the analysis of video scene structure and detection of visual attention. For example, Rui et al. [11] and Yeung et al. [12] both presented methods to group video shots and used finite state machine to incorporate audio cues for scene change detection. Since these approaches are either bottom-up or top-down, they are difficult to achieve the global optimization result. Ngo et al. [13] solved this problem by adopting normalized cut on a graph model of video shots. Our work improves their method by counting on audio similarity between shots. Zhai and Shah [14] provided a method for visual attention detection using both spatial and temporal cues. Daniel and Chen [15] visualized video sequences with volume visualization techniques. Goldman et al. [7] presented a schematic storyboard for visualizing a short video sequence and provided a variety of visual languages to describe motions in the video shot. Although this method was not suitable for exploring relations of scenes in a long video sequence, their definition of visual languages inspires our work.

Our Visual Storylines approach first clusters video shots according to both visual and audio data to form semantic video segments which we call sub-stories. The storylines are revealed by their similarities. Next, it calculates and selects the most important background, foreground and character information to composite sub-story presenters. A multi-level storyline visualization method that optimizes information layout is designed to visualize both abstract story relationships and important video segments. The details are introduced in the following two sections.

\section{Automatic Storyline Extraction}

It is necessary to extract the storylines from a video sequence before generating any type of video summaries. Automatic approaches are desirable, especially for tasks like video previewing where no user interaction is allowed. We achieve an automatic storyline extraction method through segmenting a video into multiple sets of shot sequences and measuring their relationships. Our approach considers both visual and audio features to achieve a meaningful storyline extraction.

Our storyline is defined as important paths in a weighted undirected graph of sub-stories (video segments). To generate a meaningful storyline, it is crucial to segment a video into a suitable number of video segments, which are sets of video 
shots. A shot is a continuous strip of motion picture film that 206 runs for an uninterrupted period of time. Since shots are gen-207 erally filmed with a single camera, a long video sequence may 20 contain a large number of short video shots. These video shot-209 $\mathrm{s}$ can assist us to understand video contents; however, they $\mathrm{do}_{210}$ not reflect the semantic segmentation of original videos well.

Therefore, they should be clustered as meaningful segments, 211 which are called video events.

Automatic shot clustering is a very challenging problem $\left[11,{ }^{212}\right.$ $12,13]$, as in many movie sequences, several characters talk al- ${ }^{213}$ ternatively under similar scenes or scenes may change greatly ${ }^{214}$ while a character is giving a speech. Previously, Rui et al. [11] and Yeung et al. [12] presented methods to group video shots by using thresholds to decide whether a shot should belong to ${ }^{216}$ an existing group. Since a single threshold is usually not robust enough for a whole sequence, these approaches may lead ${ }^{217}$ to over segmentation. Ngo et al. [13] used normalized cut to cluster the shots. In their work, the similarities between shots 218 contain the color and temporal information. However, none of the existing approaches are robust for movie sequences.

We believe that combining both visual and audio features ${ }_{219}$ of a video sequence can improve the results of shot clustering, leading to more meaningful segmentations for visual storylines. Figure 1 illustrates our video shot clustering algorithm, ${ }_{220}$ where we integrate several important video features to cluster video shots and calculate their relations. Although audio features have been utilized in video analysis $[16,17,18]$, we are the first to use it as features for graph modeling of video shot ${ }^{221}$ clustering.

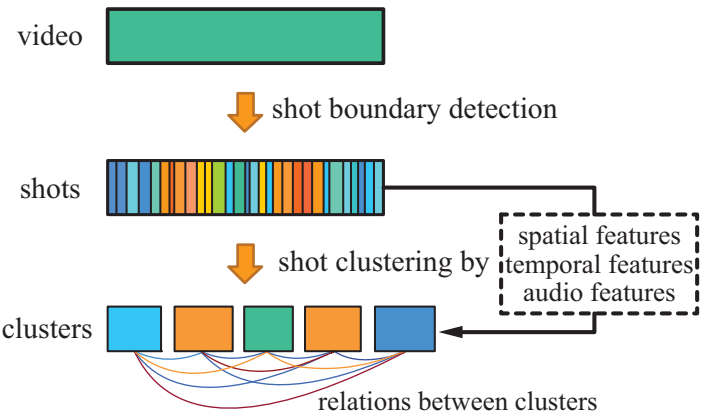

Figure 1: Our video shot clustering algorithm combines both visual and audio features to generate a meaningful storyline.

Specifically, our shot clustering algorithm integrates the fol- ${ }^{236}$ lowing visual and audio features: shot color similarity, shot au- ${ }^{237}$ dio similarity, and temporal attraction between shots. Shots are obtained using the approach proposed in [19], which can handle complex scene transitions, such as hard cut, fade and dissolve. The color similarity and temporal attraction is defined the same way as in [11], and the shot audio similarity is defined ${ }_{243}^{242}$ as an MFCC feature distance[20]. The Mel-frequency cepstral coefficients (MFCC) derived from a signal of short audio clip ${ }_{245}$ approximate the human auditory system's response more closely than the linearly-spaced frequency bands used in the normal cepstrum. It can be used as a good audio similarity measure for speaker diarisation. For each shot, we calculate the mean ${ }^{247}$ vector and covariance matrix of all the MFCC feature vectors in the shot, the audio similarity of two shot is then defined as one minus the Mahalanobis distance between the shots.

Thus, we define the overall similarity between two shots $\mathrm{x}$ and $\mathrm{y}$ as:

$$
\operatorname{ShtSim} \operatorname{im}_{x, y}=A t t r_{x, y} \times\left(W_{C} * \operatorname{Sim} C_{x, y}+W_{A} * \operatorname{Sim} A_{x, y}\right)
$$

where $A t t r_{x, y}$ is temporal attraction between shots, $W_{C}$ and $W_{A}$ are the weights for color and audio measures $\operatorname{Sim} C_{x, y}$ and $\operatorname{Sim} A_{x, y}$. Since we have the observation that larger similarity is more reliable, we define the weights as follows:

$$
W_{C}=\frac{\omega_{c}}{\omega_{c}+\omega_{a}}, W_{A}=\frac{\omega_{a}}{\omega_{c}+\omega_{a}},
$$

where

$$
\begin{aligned}
& \omega_{c}(x, y)=\left\{\begin{array}{ll}
e^{\lambda_{c}(x, y)} & \text { if } \operatorname{Sim} C_{x, y}>\mu_{c}+\frac{\sigma_{c}}{2} \\
e^{-1} & \text { otherwise }
\end{array},\right. \\
& \omega_{a}(x, y)=\left\{\begin{array}{ll}
e^{\lambda_{a}(x, y)} & \text { if } \operatorname{Sim} A_{x, y}>\mu_{a}+\frac{\sigma_{a}}{2} \\
e^{-1} & \text { otherwise }
\end{array},\right. \\
& \lambda_{c}(x, y)=-\frac{\left(1-\operatorname{Sim} C_{x, y}\right)^{2}}{\left(1-\mu_{c}-\frac{\sigma_{c}}{2}\right)^{2}}, \\
& \lambda_{a}(x, y)=-\frac{\left(1-\operatorname{Sim} A_{x, y}\right)^{2}}{\left(1-\mu_{a}-\frac{\sigma_{a}}{2}\right)^{2}} .
\end{aligned}
$$

$\mu_{c}$ and $\sigma_{c}$ are the mean and variance of color similarities, $\mu_{a}$ and $\sigma_{a}$ are the mean and variance of audio similarities.

After calculating pairwise similarities, we build weighted undirected graph and adopt normalized cut to cluster the shots. An adaptive threshold is used for termination of recursively partition as in [13]. The incorporation of audio features improves the clustering result. For example, when cluster the movie sequence in Figure 5(a), the second sub-story (represented in the upright corner of the result image) has an outdoor/indoor change, using similarity defined in [13] will improperly partition it to two cluster due to the large appearance change, but since the same character gives speech, the audio similarity is relatively large. Therefore, it gives a more semantic clustering by our similarity measure.

We use each cluster to represent a sub-story. We denote clusters as $S=\left\{S u b-\right.$ story $_{1}, S u b-$ story $_{2}, \ldots, S u b-$ story $\left._{m}\right\}$. Those sub-stories are usually not independent to each other, especially in professionally edited movies. Some sub-stories may be strongly related although they are not adjacent. For example, some movies often contain more than one story thread and different sub-stories occurred at different locations synchronously. To demonstrate this, filmmakers may cut two stories to multiple sub-stories and depict them alternately. To capture this important information, we calculate the relations between two substories. They are defined as follows:

$$
\begin{aligned}
E R_{i, j} & =W_{C} * A v g_{x \in E_{i}, y \in E_{j}} \operatorname{Sim}_{x, y} \\
& +W_{A} * A v g_{x \in E_{i}, y \in E_{j}} \operatorname{Sim}_{x, y}
\end{aligned}
$$


To handle the situation that some shots are mis-clustered, $\mathrm{we}_{300}$ empirical throw first and last 5 shots in a sub-story when calcu-30 lating the average above. We further check all the shot cluster-302 ing results generated in our paper. The video events with larger ${ }_{303}$ similarity values are viewed as being more related. We will integrate the relation information during the generation process ${ }_{304}$ of visual storylines in Section 4.

In all five video sequences, we manually labeled 43 story $^{305}$ cuts, the shot clustering with audio similarity provided 33 correct story cuts, while it reduced to 21 without audio similarity ("correct" means a story cut is detected within a distance of $5^{300}$ shots from ground truth). This proves the use of audio similari- ${ }_{310}$ ty greatly increases the accuracy of shot clustering.

\section{Generation of Visual Storylines}

With the extracted storylines, we further visualize a movie ${ }^{314}$ sequence in a new type of static visualization. This is achieved ${ }^{315}$ with a multi-level visual storyline approach, which selects and ${ }^{316}$ synthesizes important story segments according to their rela- ${ }^{317}$ tionships in a storyline. Our approach also integrates image ${ }^{318}$ and information synthesis techniques to produce both semantic ${ }^{319}$ and visual appealing results.

Previously, static summarization of a video is usually ${ }^{321}$ achieved by finding a keyframe from the sequence $[3,1,4]$ or $^{322}$ a ROI (region of interest) from the keyframe [2, 5]. Obvious- ${ }^{323}$ ly, one single keyframe or ROI is insufficient to represent many ${ }^{324}$ important information of a story, such as time, location, charac- ${ }^{325}$ ters and occurrence. Simply "stacking" all the images together, ${ }_{326}$ like "VideoCollage", is still not enough to reveal a storyline or ${ }_{327}$ roles of different characters due to lack of relationships and em- ${ }_{328}$ phasis.

Our design of the visual storyline approach is based on the ${ }_{330}$ observation that complicated stories are usually consists of multiple simple stories; while simple stories are only involved of several key factors, such as characters and locations. General- ${ }_{333}$ ly, while commercial movies contain multiple sub-stories, the ${ }_{334}$ major storylines are rather straightforward. Therefore, we can design a visual storyline as an automatic poster to visualize various movies.

For handling complicated storylines, such as commercial movies, a multi-level approach is necessary to visualize various movies because of the following reasons.

- First, since one still visualization can only provide a lim- ${ }^{33}$ ited amount of information, we need to control the details ${ }^{339}$ of visual storylines, so that they are presented at a suitable ${ }^{340}$ scale for viewers to observe.

- Second, it is important to describe major events and main ${ }^{343}$ characters instead of details that are only relevant to some ${ }^{344}$ short sub-stories. Therefore, we always need to include ${ }^{345}$ the top levels of storylines and generate visual summaries ${ }^{346}$ at different scales.

We have developed several methods to synthesize image and ${ }_{349}$ information collected from a video sequence. The following ${ }_{350}$ first introduces how to extract essential image segments by selecting background and foreground key elements, then describe our design of sub-story presenter, storyline layout and storyline visualization.

\subsection{Background Image Selection}

This step aims to find a frame which can best describe the location (or background) of a sub-story. Typically, it should be an image with the largest scene in the video sequence. Although detecting the scale from a single image is still a very hard problem in the areas of computer vision and machine learning, we can simplify this problem according to several assumptions summarized based on our observations:

Shots containing scenes of larger scales usually have smoother temporal and spatial optical flow fields. This is because these background scenes are usually demonstrated by static or slow moving cameras. In this case, if the optical flow fields indicate a zooming-in or zooming-out transition, the first or the last frame should be selected respectively since they represent scenes of largest scale.

We can remove the frames with good respondence to face detection to avoid the violation of characters' feature shots, as they are not likely to be background scene.

Very often, a shot containing this kind of frames appears at the beginning of the video sequence which is called establishing shot. The establishing shots mostly happen within first three shots of a sub-story.

Therefore, we can detect the image with the largest scale automatically using additional information collected from a video sequence. We run a dense optical flow calculation [21] and face detection algorithms [22] through the video sequence and discard shots with stable face detection respondence. The remaining shots are sorted in the ascending order of optical flow discontinuity defined as follow.

Optical flow discontinuity for $S$ hot ${ }_{i}$ from a video event ( $i$ is shot index in the video event):

$$
\operatorname{Discont}(i)=\frac{1}{n u m F r m_{i}} * \sum_{j=1}^{\operatorname{numFrm}_{i}-1}\left(D s c S_{j}+D s c T_{j}\right)
$$

Here, numFrm $_{i}$ is the frame number of $\operatorname{Shot}_{i}, \operatorname{DscS}_{j}$ is spatial optical flow discontinuity of frame $\mathrm{j}$, and $D s c T_{j}$ is temporal optical flow discontinuity between frame $\mathrm{j}$ and $\mathrm{j}+1$. They are measured the same way as in [21].

After sorting by this discontinuity value, a proper frame from each of the top ten shots is selected (due to zooming order) as the background candidate of a video sequence. To achieve this, we run a camera zoom detection for the shot according to [23], and choose the frame with smallest zoom value. We sequentially check the selected ten frames, if any of them belongs to the first three (in temporal) shots of the video sequence, it will be chosen as the background image of sub-story, as it has a large chance to be the establishing shot. Otherwise we just choose the one ranks first. A selected background image is demonstrated in the top-left corner of Figure 2. 


\subsection{Foreground ROIs Selection}

There are three kinds of objects that are good candidates of ${ }^{404}$ foreground regions of interest (ROIs) for drawing visual atten- ${ }^{405}$ tions:

Character faces. Characters often play major roles in many 408 commercial movies, where more than half of the frames con-409 taining human characters.

Objects with different motion from the background often draw ${ }^{411}$ temporal attentions.

Objects with high contrast to the background often draw spa- ${ }^{413}$ tial attentions.

Therefore, we propose a method that integrates the detection ${ }_{41}$ algorithms of human faces and spatiotemporal attentions. $\mathrm{We}_{417}$ reuse the per frame face detection result from Section 4.1 and $_{418}$ only preserve those stably detected in temporal space (detected ${ }_{419}$ in continuous 5 frames). Then, we define a face-aware spa-420 tiotemporal saliency map for each frame as:

$$
\operatorname{Sal}(I)=\kappa_{T} \times \operatorname{SalT}(I)+\kappa_{S} \times \operatorname{Sal} S(I)+\kappa_{F} \times \operatorname{SalF}(I),
$$

Here, the spatiotemporal terms are exactly the same as in [14], ${ }^{424}$ though more advanced approach such as [24] could also be ${ }^{425}$ used. We add the face detection result to the saliency map with ${ }^{426}$ the last factor. Specifically, for pixels falling in the detected ${ }^{427}$ face regions, we set its saliency value $S a l F(I)$ as 1 , or zero oth- ${ }^{428}$ erwise. $\kappa_{F}$ is the weight for $\operatorname{SalF}(I)$. Whitout violating the dy- ${ }^{429}$ namic model fusion (which means the weights are dynamically ${ }^{430}$ changed with the statistic value of $\operatorname{SalT}(I)$ ), we set $\kappa_{F}=\kappa_{S}$.

Next, we automatically select ROIs for each video sequence. ${ }^{432}$ To prevent duplicate object selection, we restrict that only one ${ }^{433}$ frame can be used for ROI selection in each shot. This frame is the one with the largest saliency value in the shot. Then for ${ }_{434}$ a new selected ROI, we check the difference between its local histogram and those of existed ROIs. If it is smaller than a 435 threshold (0.1 Chi-square distance), only the one with the larger 436 saliency value will be preserved. Those ROIs are then sorted by 437 their saliency value per pixel. Different kinds of selected ROIs438 are demonstrated in Figure 2.

\subsection{Sub-story Presenter}

We design a method to generate a static poster for present ${ }^{442}$ ing simple sub-stories. Our approach is inspired by popular ${ }^{443}$ commercial movie posters, which usually have a large stylized ${ }^{444}$ background and featured character portraits, along with multiple (relative smaller) most representative film shots. This lay-445 ered representation not only induces the user to focus on the most important information, but also provides state-of-the-art ${ }_{446}$ visual appearance.

Our sub-story presenter contains at least four layers. $\mathrm{The}_{448}$ bottom layer is the background image frame extracted in sec- ${ }_{449}$ tion 4.1. The layer next to bottom contains ROIs with no face detected, while other layers are composed of other ROIs ex- ${ }_{450}$ tracted in section 4.2. The higher layer contains ROIs with higher order, i.e. higher saliency values. We use a greedy algo-451 rithm to calculate the layout, as illustrated in Figure 2.
We start from the bottom layer, i.e. the background image. We initialize the global saliency map with the saliency map of background image. Then we add each layer overlapping on the presenter from the lowest layer to the top layer. For each layer, we add ROIs from the one with the highest saliency value to the lowest. For each ROI, we first resize it according to its saliency degree, then search for a position that minimizes the global saliency value of the presenter covered by the ROI. After adding a new ROI, global saliency map is updated by replacing covered region's saliency with newly added ROI's.

In this progress, we use a threshold $\varphi$, which we called level of detail controller, to control the amount of presented ROIs. That means, when adding a new ROI, every objects in the presenter (including background image) must preserve at least $\varphi$ portion of its original saliency value in the global saliency map (detected face region has the exception that it should never be covered, to prevent half face). When this is violated, the ROI with least saliency will be removed from the presenter, and recalculate the layout. With this "LOD" control, when the video sequence we represented becomes more complicated, we can ensure each presented part still provides sufficient information.

After adding each layer, we use graph cut to solve labeling problem followed by $\alpha$-poisson image blending [25]. To emphasize the importance of foreground objects, we stylize each layer as shown in Figure 2. We compute the average hue value of background image, use this value to tint each layer, and lower layers will be tinted by larger proportions. Figure 3 shows six basic event presenters synthesized by our approach. They are able to represent most important information of the video even$\mathrm{t}$ such as locations, characters, and also preserve the original video style.

\subsection{Storyline Geometric Layout}

Now the remaining problem is how to arrange sub-story presenters on the final visual storylines to reveal their relationships. We prefer to preserve the style of movie posters, so that visual storylines are intuitive for general users to understand. Here, we present an automatic algorithm of storyline geometric layout through utilizing all the extracted information from video analysis.

Given $n$ sub-story presenters $\left\{R_{1}, R_{2}, \ldots, R_{n}\right\}$ for $n$ sub-stories and their relations, and a canvas of size $l \times m$, we first resize all the sub-story presenters:

$$
\operatorname{size}\left(R_{i}\right)=\max \left(0.25, \frac{L\left(R_{i}\right)}{L_{\max }}\right) \times \frac{l * m}{1.5 n},
$$

where $L\left(R_{i}\right)$ is the length (in frame) of the $S u b-s t o r y_{i}, L_{\text {max }}$ is the maximal duration of all the sub-stories. Let $\left(x_{i}, y_{i}\right)$ denotes the shift vector of the sub-story presenters $R_{i}$ on canvas, then we minimize the following energy function:

$$
E=E_{\text {ovl }}+w_{\text {sal }} * E_{\text {sal }}+w_{\text {rela }} * E_{\text {rela }}+w_{\text {time }} * E_{\text {time }},
$$

overlay term $E_{o v l}=-A_{o v l}$ is the negative of the overlay area of all the basic event presenters on the canvas; Saliency cost $E_{\text {sal }}$ 

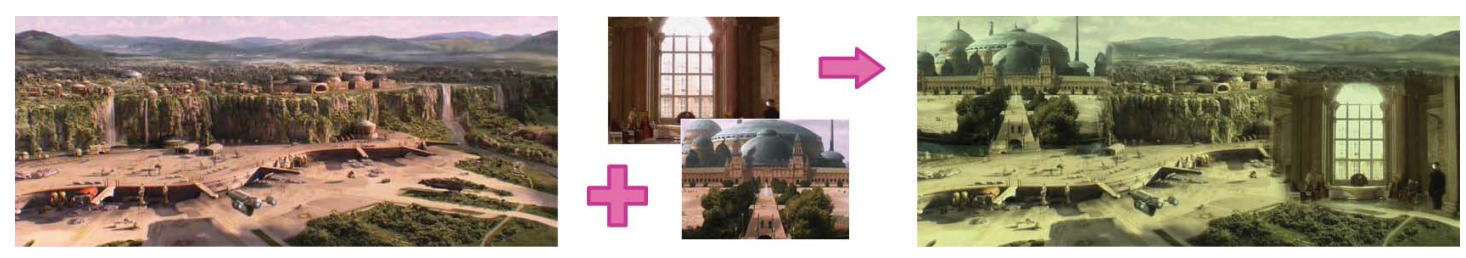

Ґ : saliency sensitive layout

$\Rightarrow$ : graph cut, alpha blending and stylization
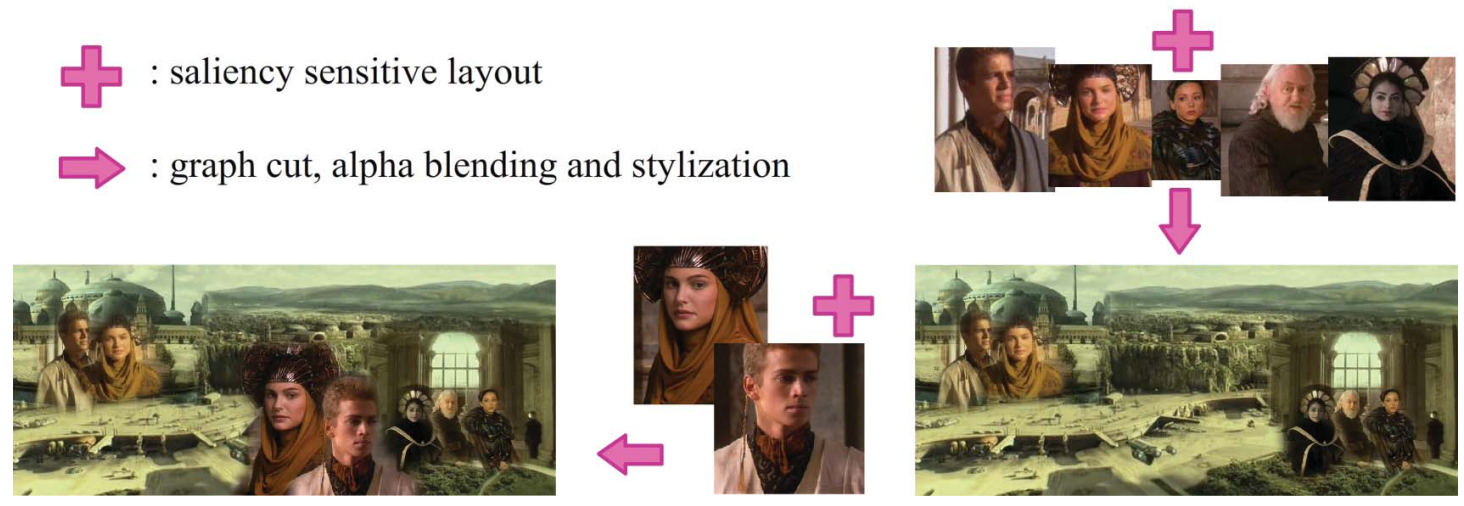

Figure 2: Synthesis progress of the sub-story presenter.


Figure 3: Storyline geometric layout. The right figure is a synthesized visual storyline for a video sequence of 30 min, which is clustered to 10 sub-stories. For limited spaces, the figures on the left show six sub-story presenters.

is negative saliency value of composed saliency map; Relation ${ }_{466}$ term is defined as:

$$
E_{\text {rela }}=\sum_{i=0}^{n} \sum_{j=i+1}^{i+3}\left(\operatorname{Dist}(i, j)-\frac{\sqrt{l m}\left(E R_{\max }-E R_{i, j}\right)}{E R_{\max }-E R_{\min }}\right)^{2},
$$

where $E R_{i, j}, E R_{\max }$ and $E R_{\min }$ are relationships measured be-472 tween $S u b-$ story $_{i}$ and $S u b-$ story $_{j}$, maximal relationship and $_{473}$ minimal relationship respectively. $\operatorname{Dist}(i, j)$ is the distance be- ${ }_{474}$ tween the centers of two basic event presenters. This term at- 475 tempts to position sub-story presenters with larger relation clos- ${ }_{476}$ er to each other in $\mathrm{x}$ coordinate; Temporal order term is defined ${ }_{477}$ as:

$$
E_{\text {time }}=\sum_{i=0}^{n-1} \delta_{i}
$$

where

$$
\delta_{i}= \begin{cases}0 & \text { if } y_{i}+\epsilon<y_{i+1}<y_{i}+h_{i}-\epsilon \\ 1 & \text { otherwise }\end{cases}
$$

$h_{i}$ is the height of resized $R_{i}$, and $\epsilon=30$. This term attempts to a sub-story presenter, when each new presenter is put in, the algorithm calculates its position that minimize the current energy function. As this method can not ensure that all pixels are covered, we can choose those obsoleted ROIs from adjacent basic event presenters to fill the hole. An alternative is to adopt the layout optimization method described [26] in Overlapped region will be labeled by graph cut and $\alpha$-poisson image blending. Since overlapping may cause the violation of LOD control, it is necessary to recalculate the layout for sub-story presenters. Figure 3 shows the events layout and the LOD control effect. It shows when the represented video sequence becomes complicated, our results will not be cluttered as other methods while 
still provide essential video information.

\subsection{Storyline Visualization}

The final visual storylines are enriched with a sequence of arrow shapes to represent key storylines. This is achieved by building a storyline graph, which uses video sub-stories as nodes. For two adjacent video sub-stories in the visual storylines, if the relationship between them is larger than a threshold, we add an edge in between. After traversing all the nodes, circles will be cut off at the edge between two nodes with largest ${ }_{517}$ temporal distance. Then, each branch in this acyclic graph rep- ${ }_{518}$ resents a story line. We add an arrow around the intersection ${ }_{519}$ location between any two connected sub-story presenters with ${ }_{520}$ the restriction that no ROI is covered. The directions of arrows $\mathrm{S}_{521}$ illustrating the same storyline are calculated according to a $\mathrm{B}_{-522}$ spline, which is generated by connecting all the arrow centers ${ }_{523}$ and saliency-weighted centers of involved sub-story presenters ${ }_{524}$ on this storyline. This can produce a most smooth and natu-525 ral illustration from the storyline. The arrow bottom is reduced ${ }_{526}$ to disappear among the previous event to emphasize the direc-527 tion of storylines. Different storylines are distinguished by the ${ }_{528}$ colors of arrows.

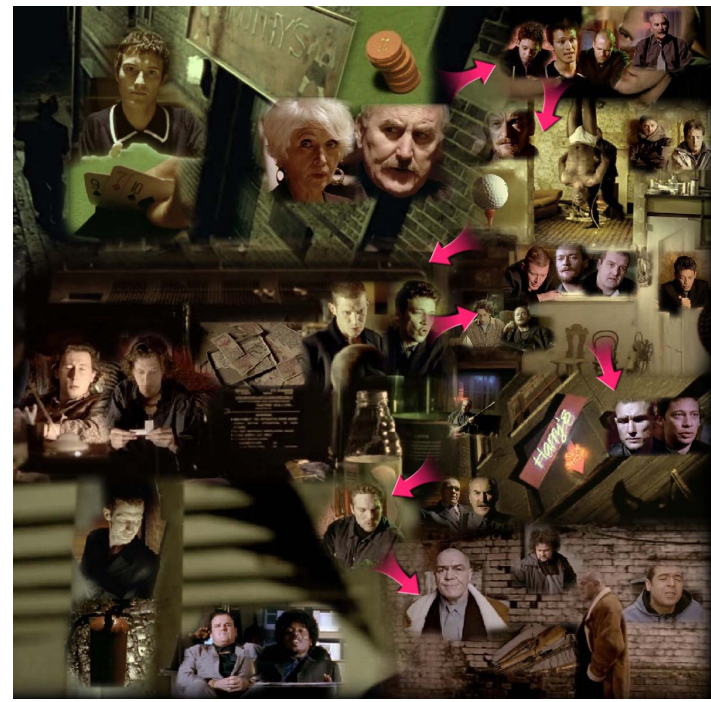

Figure 4: A failed case of our system when representing 25 minutes video547 sequence from the commercial movie Lock, Stock, and Two Smoking Barrels. ${ }_{548}$ User studies show this summary can't reveal the true storylines of the movie sequence.

\section{Experiments and Evaluations}

\subsection{Experimental Results}

Figure 5 shows example results of visual storylines. Their computation times on a Core 2 Duo 2.0Ghz machine and LOD $_{557}$ thresholds $(\varphi)$ are shown in Table 1.

The video sequence used in Figure 5(a) is a classic movie ${ }_{558}^{558}$ clip that features two scenes (different locations and characters) alternately. Our approach successfully extracts the two story-560 lines. Note that the movie title in the result is a manually added ${ }_{561}$ ROI, which replaces the correspondence part in Figure 3.

\begin{tabular}{|l|c|c|c|}
\hline Video clip & Length & Time cost & $\varphi$ \\
\hline Fig.5(a): StarWars & $30 \mathrm{~min}$ & $125 \mathrm{~min}$ & $40 \%$ \\
\hline Fig.5(b): Lost & $20 \mathrm{~min}$ & $80 \mathrm{~min}$ & $60 \%$ \\
\hline Fig.5(c): Heroes & $22 \mathrm{~min}$ & $90 \mathrm{~min}$ & $70 \%$ \\
\hline Fig.5(d): Crazy & $15 \mathrm{~min}$ & $62 \mathrm{~min}$ & $40 \%$ \\
\hline
\end{tabular}

Table 1: Computation times for each representation result.

Figure 5(b) and (c) visualize two fast-paced TV programs. They both have multiple storylines progressed together, which is a popular technique in modern TV-series. Our approach extracts the main storylines for each program. Although one storyline (threaded by the pink arrows) in (c) has merged two semantic scenes together due to the very similar scene presences, our later user studies show that viewers can still understand the plot with our visual stories. Note user can adjust LOD threshold $\varphi$ to generate multi-level results. The multi-level visual storylines generated by different thresholds for Figure 5(b) and (c) are demonstrated in the supplementary file.

Figure 5(d) visualizes a movie clip that alternately features two groups of characters, which finally meet each other. Our visual storylines reveal this important feature with two merging storylines.

In summary, our approach of visual storylines is suitable for visualizing the movie scenes with salient appearance attribute, like desert, meadow, sky and other outdoor scenes, or indoor scenes with artistic stylized illumination. The changes of characters may also help the system distinguishing different scenes.

One failed case is shown in Figure 4. Commercial movie Lock, Stock, and Two Smoking Barrels is famous for its fast scene changes and techniques of expressing multiple storylines. In this movie, most scenes in those different storylines are indoor scenes with indistinguishable color models. What's more, character groups in different scenes have complex interaction with each other. Therefore, our approach cannot extract correct storylines. The extracted storylines are with respect to the temporal order of the sub-story presenter.

\subsection{User Studies and Discussion}

We have designed three user studies to evaluate our approach. The first user study is designed to check the aesthetic measure and representative measure comparing with other methods.

Twenty subjects are invited for this user study, including fourteen graduate students and six undergraduate students (majoring in computer science, architecture and art) who are unaware of our system. Four kinds of video summaries (Booklet, Pictorial, Video Collage and Visual Storylines) are created for sequences shown in Figure 5. After watching the video sequences, users have been asked to answer the following questions with scale 1 (definitely no) to 5 (definitely yes), as used in $[25,5]$. Here we list our questions and provide the average scores and standard deviances for each method after their names.

- Are you satisfied with this summary in general?

Visual Storylines(4.10, 0.62), Video Collage(3.50, 0.67), Pictorial(2.30, 0.90), Booklet(2.45, 0.97) 
- Do you believe that this result can represent the whole 617 video sequence?

Visual Storylines(4.20, 0.68), Video Collage(3.65, 0.65),619 Pictorial(3.30, 0.64), Booklet(3.15, 0.57)

- Do you believe this presentation is compact? Visual Storylines(4.00, 0.71), Video Collage(3.90, 0.70), Pictorial(2.60, 0.49), Booklet(2.35, 0.57)

- Would you like to use this result as a poster of the video? ${ }^{625}$ Visual Storylines $(4.65,0.48)$, Video Collage $(3.70,0.71){ }^{626}$ Pictorial(1.4), Booklet(3.1)

627

- Do you believe that this presentation produces the correct629 storylines?

Visual Storylines(4.85, 0.36), Video Collage(2.25, 0.70), 631 Pictorial(2.5, 0.74), Booklet(1.75, 0.83)

The results demonstrate that our approach achieves the high-634 est scores in all the categories; therefore, it is the most repre-635 sentative and visual appealing summary among these four ap- 636 proaches. This also shows that Visual Storylines is the only ${ }_{637}$ approach that extracts and visualizes video storylines.

638

The other two user studies are designed to evaluate if our 639 results can help user quickly grasp major storylines without ${ }_{640}$ watching a video. Note that it's generally very difficult for 641 someone to understand the semantic storylines of a movie or ${ }_{642}$ TV program from a single image without knowing any contexts.643 In the second user study, subjects are asked to watch some video ${ }_{644}$ clips related to the test video. Specifically, fifteen more subject- 645 $\mathrm{S}$ are invited and confirmed that they have not seen any of the ${ }_{646}$ movies or TV programs appeared in Figure 4 and Figure 5 be-647 fore. Ten of them are assigned to "test group", the other five ${ }_{648}$ were assigned to "evaluation group". We showed the test group 649 the five movies/TV programs used in our paper but skipped the ${ }_{650}$ parts that used to generate the video summaries. The evaluation ${ }_{651}$ group was allowed to watch the full movies or TV program-652 s. Then in the test group, half of the subjects were provided ${ }_{653}$ with five summaries generated by our method, while the other ${ }_{654}$ half were provided with five summaries generated by "Video 655 Collage" (since it's most competitive in the first user study). .656 Then these ten subjects were asked to write text summaries for ${ }_{657}$ the five video clips they missed. These text summaries were ${ }_{658}$ shown to the evaluation group, and evaluated from 1 (very bad b59 $_{69}$ summaries) to 5 (very good summaries). The average score for 660 each video by different methods is shown in Table 2 .

In the third user study, we invited ten more subjects. They662 were asked to read text synopsis for the five videos tested in our 663 paper. They were also provided with the summaries (Visual S-664 torylines for half, Video Collage for the other half). Then they ${ }_{665}$ were asked to circle the corresponding regions in the summaries 666 for some previously marked keywords in the synopsis, which ${ }_{667}$ included locations, objects and character names. We manually ${ }_{668}$ checked the correctly circled regions and list the result in Ta-669 ble 2 .

Table 2 shows when viewers know the context of the video,671 for example the main characters and their relationship, the pre-672 ceding and succeeding stories, they can easily understand the ${ }_{673}$ stories with our visual storylines. It also shows viewers can quickly establish correct connections between the text synopsis and our summaries. Note the two statistic results of Lock, $S$ tock, and Two Smoking Barrels are lower than 3 and lower than $60 \%$.

The user studies reveal two potential applications for our approach. First, if a viewer misses an Episode of TV show or a part of the movie, visual storylines can be synthesized to help the viewer quickly to grasp the missing information. Second, when providing our result together with the text synopsis of the video, viewers get a visual impression of the story described in the synopsis. Therefore, our automatically generated results can be easily integrated into the TV guide newspapers, movie review magazines and movie websites as illustration of the text synopsis.

Except the comparison with the methods of generating static summarization for long video sequence, we'd also like to discuss and compare with those state-of-the-art video summarization methods. As $[6,7]$ mainly focus on one or two characters and their spatial motion, their summarization is very suitable for visualizing one or several shots. On the other hand, they can't deal with long video sequences like our method. However, if we incorporate their static representations of character motion into our sub-story representation, the visual storylines can be more compact and less visually repetitive. The Video Tapestries [10] provides similar static summarization form to ours except their shot layout is purely sequential. But when the multiscale summarization is interactively viewed by the user, it can provide more information than our method. However, our static result is more suitable for traditional paper media.

Here, we discuss some limitations of our approach and possible improvements. As the failure case indicated, our approach generates limited result for indistinguishable scenes. In addition, as it selects important candidates according to low level features such as visual saliency and frequency, the visualization may still miss crucial semantic information. For example, the coffin, which plays an important role in the result collage of Lost sequence is barely recognizable. Another issue about our approach is that even with LOD control, our result may still suffer from repetitively showing main characters as in other methods. One solution, as mentioned above, is to adopt the character motion representations described in $[6,7]$, or generate motion photography in static image similar to [27]. We may also try to recognize repeating characters or foreground objects from their appearance and segmentation silhouettes by the boundary band map matching method introduced in [28]. A recently emerged candid portrait selection approach [29] which learned a model from subjective annotation could also help us to find more visual appealing character candidates. The $\alpha$-poisson image blending we adopted to composite the visualizations sometimes generates undesirable cross-fading, which could be resolved by recent developed blending methods such as hybrid blending [30] or environment-sensitive cloning [31]. Lastly, our preliminary user study could also be improved. The questions in the first user study are too general and subjective, which may bias the evaluation due to the understanding of the video sequences of each individual. The second user study is too complicatedly 


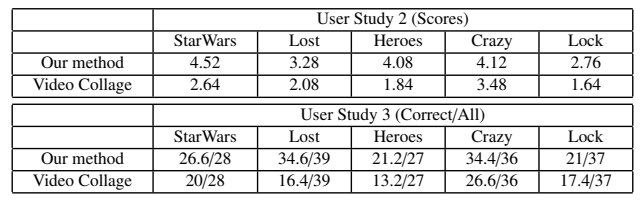

Table 2: The statistic results for user study 2 and 3

designed and may bias from the writing skill of the individual. ${ }^{735}$

\section{Conclusion}

This paper presents a multi-level visual storyline approach to ${ }^{739}$ abstract and synthesize important video information into suc- ${ }^{740}$ cinct still images. Our approach generates visually appealing ${ }_{742}$ summaries through designing and integrating techniques of au-743 tomatic video analysis and image and information synthesis $\mathrm{We}^{744}$ have also designed and performed preliminary user studies to e $-^{745}$ valuate our approach and compare with several classical video ${ }_{747}^{746}$ summary methods. The evaluation results demonstrate that our 748 visual storylines reveal more semantic information than previ- ${ }^{749}$ ous approaches, especially on preserving main storylines.

The techniques of video visualization and summary are $\mathrm{an}_{752}^{751}$ important addition to handle the enormous volume of digital 753 videos, as they allow viewers to grasp the main storylines of ${ }^{754}$ a video quickly without watching the entire video sequence, ${ }_{756}^{755}$ especially when they are familiar with the context of the video, 757 or a text synopsis is provided. With the efficiency provided by758 video visualization techniques, we believe that they can also ${ }^{759}$ be used to assist other video operations, such as browsing and ${ }_{761}^{760}$ documentation for entertainment and educational purposes.

\section{Acknowledgements}

This work was supported by the National Basic Research 76 Project of China (Project Number 2011CB302205), the Natural ${ }^{766}$ Science Foundation of China(Project Number $61120106007{ }^{767}$ 61033012).

\section{References}

[1] Hua XS, Li S, zhang HJ. Video booklet. ICME 2005;0:4-5. doi: http://doi.ieeecomputersociety.org/10.1109/ICME.2005.1521392.

[2] Chiu P, Girgensohn A, Liu Q. Stained-glass visualization for highly con- ${ }^{774}$ densed video summaries. IEEE International Conference on Multimedia ${ }_{776}$ and Expo 2004;3:2059-62.

[3] Yeung M, Yeo BL. Video visualization for compact presentation and fast ${ }_{777}^{777}$ browsing of pictorial content. IEEE Transactions on Circuits and Systems ${ }_{779}$ for Video Technology 1997;7(5):771-85. doi:10.1109/76.633496.

[4] Ma YF, Zhang HJ. Video snapshot: A bird view of video sequence. ${ }_{78}$ Proceedings of the 11th International Multimedia Modelling Conference, ${ }_{782}$ 2005::94-101doi:10.1109/MMMC.2005.71.

[5] Wang T, Mei T, Hua XS, Liu XL, Zhou HQ. Video collage: A novel ${ }_{783}^{783}$ presentation of video sequence. IEEE International Conference on Mul- ${ }_{785}$ timedia and Expo 2007;:1479-82doi:10.1109/ICME.2007.4284941.

[6] Correa CD, Ma KL. Dynamic video narratives. ACM Trans Graph ${ }_{787}^{786}$ 2010;29:88-9. doi:http://doi.acm.org/10.1145/1778765.1778825.

[7] Goldman DB, Curless B, Seitz SM, Salesin D. Schematic storyboarding ${ }^{788}$ for video visualization and editing. ACM Transactions on Graphics (Proc ${ }_{790}$ SIGGRAPH) 2006;25(3).

[8] Money AG, Agius H. Video summarisation: A conceptual framework and survey of the state of the art. Journal of Visual Com- ${ }_{793}$ munication and Image Representation 2008;19(2):121 -43. doi:DOI: ${ }_{794}$ 10.1016/j.jvcir.2007.04.002.

[9] Huet B, Merialdo B. Automatic video summarization. In: Hammoud RI, editor. Interactive Video. Signals and Communication Technology; Springer Berlin Heidelberg. ISBN 978-3-540-33215-2; 2006, p. 27-42.
[10] Barnes C, Goldman DB, Shechtman E, Finkelstein A. Video tapestries with continuous temporal zoom. ACM Trans Graph 2010;29:89-90. doi: http://doi.acm.org/10.1145/1778765.1778826.

[11] Rui Y, Huang TS, Mehrotra S. Exploring video structure beyond the shots. In: In Proc. of IEEE conf. Multimedia Computing and Systems. 1998, p. 237-40.

[12] Yeung M, Yeo BL, Liu B. Extracting story units from long programs for video browsing and navigation. Proceedings of the Third IEEE International Conference on Multimedia Computing and Systems 1996;:296305doi:10.1109/MMCS.1996.534991.

[13] Ngo CW, Ma YF, Zhang HJ. Video summarization and scene detection by graph modeling. IEEE Transactions on Circuits and Systems for Video Technology 2005;15(2):296-305. doi:10.1109/TCSVT.2004.841694.

[14] Zhai Y, Shah M. Visual attention detection in video sequences using spatiotemporal cues. In: MULTIMEDIA '06: Proceedings of the 14th annual ACM international conference on Multimedia. New York, NY, USA: ACM. ISBN 1-59593-447-2; 2006, p. 815-24. doi: http://doi.acm.org/10.1145/1180639.1180824.

[15] Daniel G, Chen M. Video visualization. In: VIS '03: Proceedings of the 14th IEEE Visualization 2003 (VIS'03). Washington, DC, USA: IEEE Computer Society. ISBN 0-7695-2030-8; 2003, p. 54-5. doi: http://dx.doi.org/10.1109/VISUAL.2003.1250401.

[16] Wang Y, Liu Z, Huang JC. Multimedia content analysis-using both audio and visual clues. IEEE Signal Processing Magazine 2000;17(6):12-36. doi:10.1109/79.888862.

[17] Sugano M, Nakajima Y, Yanagihara H. Automated mpeg audio-video summarization and description. In: Proceedings of the IEEE International Conference on Image Processing; vol. 1. 2002, p. I956-9. doi: 10.1109/ICIP.2002.1038186.

[18] He L, Sanocki E, Gupta A, Grudin J. Auto-summarization of audio-video presentations. In: 7th ACM International Conference On Multimedia. 1999, p. 489-98.

[19] Lienhart RW. Comparison of automatic shot boundary detection algorithms. In: Yeung MM, Yeo BL, Bouman CA, editors. Proc. SPIE Vol. 3656 , p. 290-301, Storage and Retrieval for Image and Video Databases VII, Minerva M. Yeung; Boon-Lock Yeo; Charles A. Bouman; Eds.; vol. 3656 of Presented at the Society of Photo-Optical Instrumentation Engineers (SPIE) Conference. 1998, p. 290-301.

[20] Rabiner L, Schafer R. Digital Processing of Speech Signals. Englewood Cliffs: Prentice Hall; 1978.

[21] Black MJ, Anandan P. The robust estimation of multiple motions: parametric and piecewise-smooth flow fields. Comput Vis Image Underst 1996;63(1):75-104. doi:http://dx.doi.org/10.1006/cviu.1996.0006.

[22] Lienhart R, Maydt J. An extended set of haar-like features for rapid object detection. In: IEEE ICIP 2002; vol. 1. 2002, p. 900-3.

[23] Wang R, Huang T. Fast camera motion analysis in mpeg domain. In: Image Processing, 1999. ICIP 99. Proceedings. 1999 International Conference on; vol. 3. 1999, p. 691-4. doi:10.1109/ICIP.1999.817204.

[24] Cheng MM, Zhang GX, Mitra NJ, Huang X, Hu SM. Global contrast based salient region detection. In: IEEE CVPR. 2011, p. 409-16.

[25] Rother C, Bordeaux L, Hamadi Y, Blake A. Autocollage. In: SIGGRAPH '06: ACM SIGGRAPH 2006 Papers. New York, NY, USA: ACM. ISBN 1-59593-364-6; 2006, p. 847-52. doi: http://doi.acm.org/10.1145/1179352.1141965.

[26] Huang H, Zhang L, Zhang HC. Image collage: Arcimboldo-like collage using internet images. ACM Transactions on Graphics 2011;30(6).

[27] Teramoto O, Park I, Igarashi T. Interactive motion photography from a single image. The Visual Computer 2010;26:1339-48. 10.1007/s00371-009-0405-6; URL http://dx.doi .org/10.1007/s00371-009-0405-6.

[28] Cheng MM, Zhang FL, Mitra NJ, Huang X, Hu SM. Repfinder: Finding approximately repeated scene elements for image editing. ACM Transactions on Graphics 2010;29(4):83:1-8.

[29] Fiss J, Agarwala A, Curless B. Candid Portrait Selection From Video. ACM Transactions on Graphics 2011;30(6).

[30] Chen T, Cheng MM, Tan P, Shamir A, Hu SM. Sketch2photo: internet image montage. ACM Transactions on Graphics 2009;28(5):124: 1-10.

[31] Zhang Y, Tong R. Environment-sensitive cloning in images. The Visual Computer 2011;27:739-48. 10.1007/s00371-011-0583-x; URL http://dx.doi.org/10.1007/s00371-011-0583-x. 




(a)

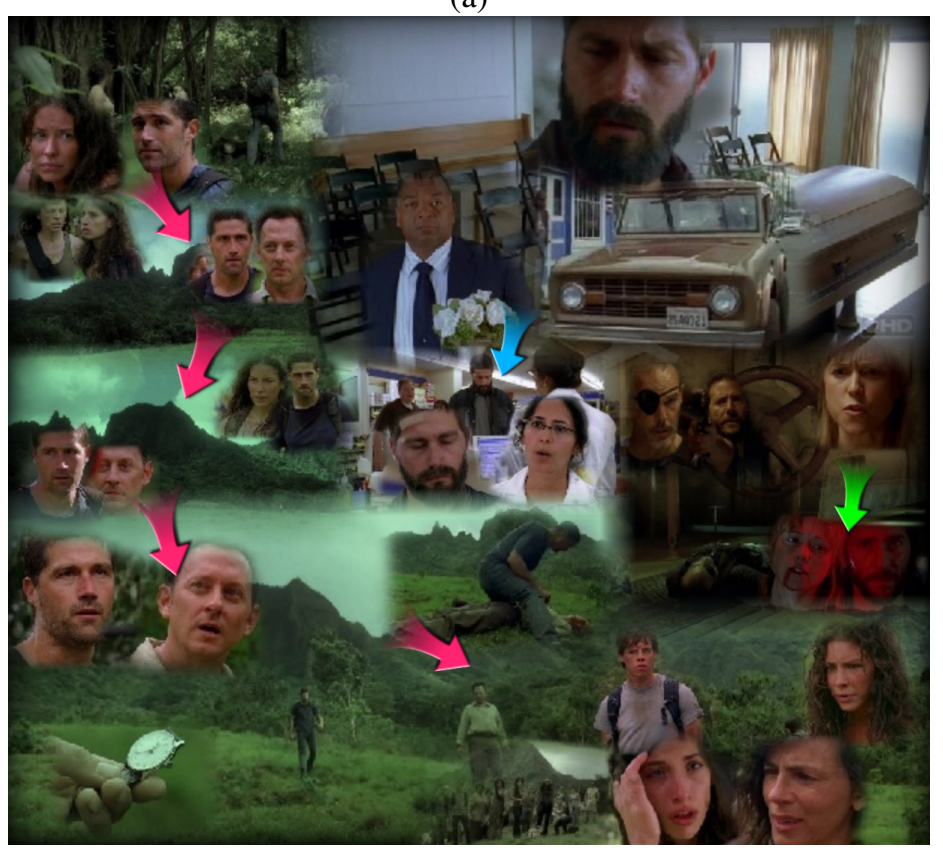

(b)



(c)

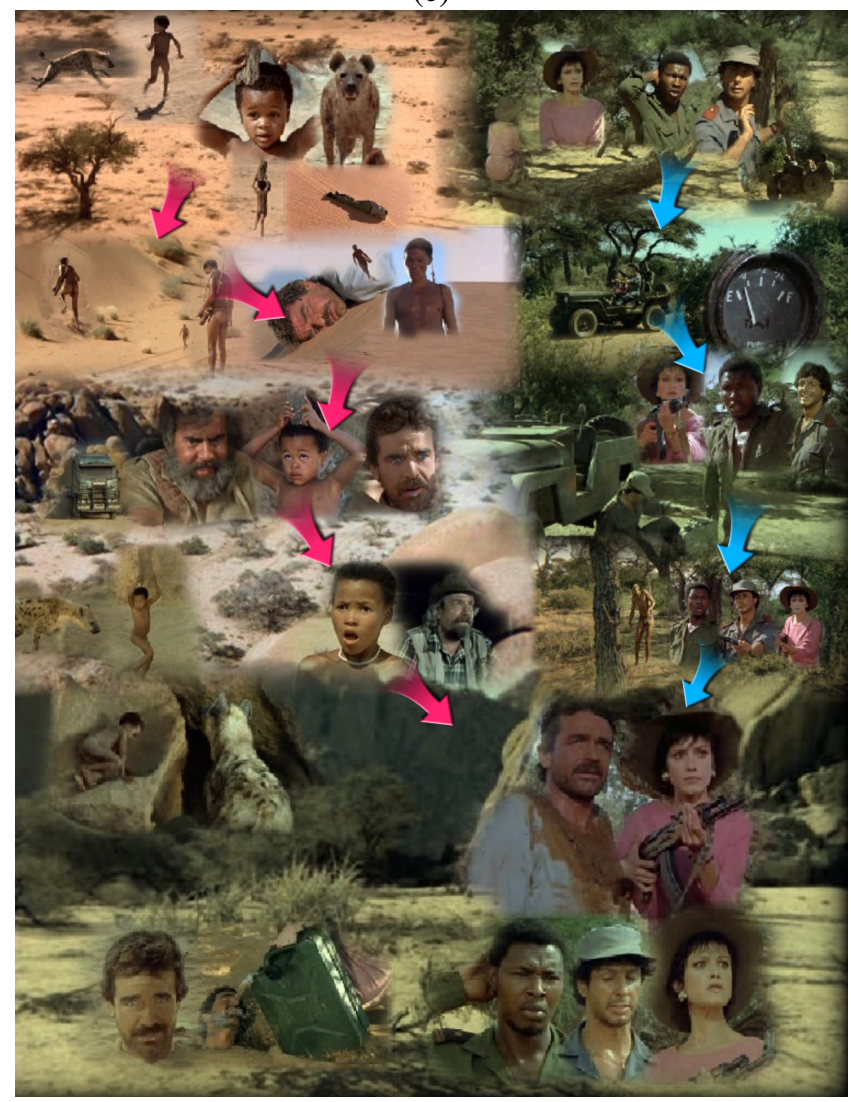

(d)

Figure 5: Visual storylines of (a) a 30 minutes sequence from the commercial movie Star Wars: Attack of the Clones, (b) a 20 minutes sequence from the TV program Lost, (c) a 30 minutes sequence from the TV program Heroes, (d) a 20 minutes sequence from the commercial movie The Gods Must Be Crazy 2. 